\title{
A new tip correction for actuator line computations
}

\author{
Da, Kaya Onur; Sørensen, Jens Nørkær
}

Published in:
Wind Energy

Link to article, DOI:

$10.1002 /$ we. 2419

Publication date:

2020

Document Version

Peer reviewed version

Link back to DTU Orbit

Citation (APA):

Da, K. O., \& Sørensen, J. N. (2020). A new tip correction for actuator line computations. Wind Energy, 23(2), 148-160. https://doi.org/10.1002/we.2419

\section{General rights}

Copyright and moral rights for the publications made accessible in the public portal are retained by the authors and/or other copyright owners and it is a condition of accessing publications that users recognise and abide by the legal requirements associated with these rights.

- Users may download and print one copy of any publication from the public portal for the purpose of private study or research.

- You may not further distribute the material or use it for any profit-making activity or commercial gain

- You may freely distribute the URL identifying the publication in the public portal

If you believe that this document breaches copyright please contact us providing details, and we will remove access to the work immediately and investigate your claim. 


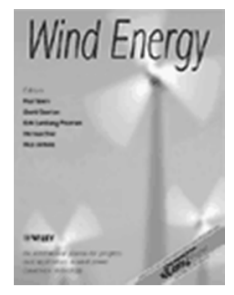

\section{A new tip correction for actuator line computations}

\begin{tabular}{|r|l|}
\hline Journal: & Wind Energy \\
\hline Manuscript ID & WE-17-0183 \\
\hline Wiley - Manuscript type: & Research Article \\
\hline Date Submitted by the Author: & $03-J u l-2017$ \\
\hline Complete List of Authors: & $\begin{array}{l}\text { Dag, Kaya; Technical University of Denmark, Wind Energy } \\
\text { Sørensen, Jens; Technical University of Denmark, Department of Wind } \\
\text { Energy }\end{array}$ \\
\hline Keywords: & $\begin{array}{l}\text { Actuator line, Tip correction, Wind turbine blade loadings, LES of wind } \\
\text { turbines }\end{array}$ \\
\hline
\end{tabular}




\title{
RESEARCH ARTICLE
}

\section{A new tip correction for actuator line computations}

\author{
Kaya O. Dag and Jens N. Sørensen \\ DTU Wind Energy, Technical University of Denmark, 2800 Kongens Lyngby, Denmark
}

\begin{abstract}
The actuator line method (ALM) is today widely used to represent wind turbine loadings in computational fluid dynamics (CFD) computations. As opposed to resolving the whole blade geometry, the methodology does not require geometry-fitted meshes, which makes it fast to apply. In ALM, tabulated airfoil data are used to determine the local blade loadings, which subsequently are projected to the CFD grid using a Gaussian smearing function. To achieve accurate blade loadings at the tip regions of the blades, the width of the projection function needs to be narrower than the local chord lengths, requiring CFD grids that are much finer than what is actually needed in order to resolve the energy containing turbulent structures of the atmospheric boundary layer (ABL). On the other hand, employing large widths of the projection function may result in too large tip loadings. Therefore, the number of grid points required to resolve the blade and the width of the projection function have to be restricted to certain minimum values if unphysical corrections are to be avoided. In this paper, we investigate the cause of the over estimated tip loadings when using coarse CFD grids, and, based on this, introduce a simple and physical consistent correction technique to rectify the problem. To validate the new correction, it is first applied on a planar wing where results are compared with the lifting-line technique. Next, the NREL 5MW and Phase VI turbines are employed to test the correction on rotors. Here the resulting blade loadings are compared to results from the blade-element momentum (BEM) method. In both cases it is found that the new correction greatly improves the results for both normal and tangential loads, and that it is possible to obtain accurate results even when using a very coarse blade resolution. Copyright (c) 2017 John Wiley \& Sons, Ltd.
\end{abstract}

\section{KEYWORDS}

actuator line, tip correction, wind turbine blade loadings, LES of wind turbines

\section{Correspondence}

DTU Wind Energy, Technical University of Denmark, 2800 Kongens Lyngby, Denmark

E-mail:kdag@dtu.dk

Received...

\section{INTRODUCTION}

The actuator line method (ALM) was developed as a numerical technique to facilitate the representation of the rotor blades in computational fluid dynamics (CFD) simulations of wind turbines [1]. The ALM is based on a blade-element approach in which body forces are introduced in the Navier-Stokes equations along lines representing the blades of the wind turbine. The body forces are obtained from tabulated airfoil data employing the local angle of attack along the blades as input. The local angle of attack is defined from the local relative velocity, which is determined at each time step while running a simulation. After having determined the local forces, they are projected from the lines representing the rotor blades 
to the CFD grid points. A main issue of the technique is that it is required to smear out the body forces in order to avoid discontinuities in the resulting force distribution. This is typically carried out by using a 3D Gaussian filtering function with a given projection width $\epsilon$, which eventually defines the thickness of the volume on which the blade forces are projected. It is known that very small $\epsilon$-values cause oscillations in the flow field, whereas large values result in inaccurate loading distributions. There has been fair amount of work on determining the optimum value of the $\epsilon$-parameter. Troldborg [2] suggested that fixing $\epsilon$ to twice the CFD grid resolution $(\Delta)$ is a good trade-off between having high accuracy and avoiding oscillations in the flow field. In the work by Jha et al. [3], it was shown that oscillations could be avoided by using a blended dissipative scheme with $\epsilon=2 \Delta$. It was also noted that a constant $\epsilon$ parameter throughout the blade-span causes too large blade tip loadings. Additionally, Jha et al. [3] introduced a new methodology to vary the $\epsilon$-parameter throughout the blade span in order to achieve a better loading distribution around the tip region. Their methodology improves the estimated loading distributions, but it also requires case-specific tuning of the parameter. In the work by Shives \& Crawford [4], the effect of the $\epsilon$-parameter and the resolution of the CFD grid was investigated for a fixed wing. For this case it was concluded that the $\epsilon$-parameter should be in the order of $1 / 4 c$, where $c$ is the chord length of the local airfoil section. Further, they stated that, in order to be consistent with the vortex line approach, the self-induction at the vortex center should be zero. Their work also showed that $\epsilon$-values smaller than $4 \Delta$ do not satisfy this condition. In a recent work by Martinez-Tossas et al. [5], a theoretical model based on the potential flow over a Joukowsky airfoil was developed to determine the optimum value of $\epsilon$. Here it was concluded that for a grid resolution larger than the chord lengtht, the choise of the scale of the smoothing-kernel is dictated by the mesh, whereas for finner discretizations a value $\epsilon=0.2 c$ should be employed, which is in fact very close to the guidelines by Shives \& Crawford [4].

Since the ALM is based on solving the three-dimensional Navier-Stokes equations around the actuator line, in principle, it is not needed to introduce a tip correction. However, when crude meshes are used there is no guarentee that the loading tends to zero at the tip, and therefore it is sometimes seen that researchers resort to the Prandtl/Glauert tip correction, which essentially is a technique for correcting the loading for finite number of blades effects in the momentum theory (see [6]). A technique for correcting the use of airfoil data near the tip was introduced by Shen et al. [7] by multiplying the lift coefficient by a function resembling the Prandtl tip-correction. This technique certainly improves the loading near the tip of the wing, but it is based on an empirical expression that may not be valid for all wing plan forms. An alternative correction to the one by Shen et al. [7] is the decamber correction introduced by Sørensen et al. [8]. This correction is based on the fact that the induction from the wake causes a curved streamline along the chord line that effectively corresponds to an additional camber of the airfoil. The correction was shown to be important for constant chord wings, but not significant for wing forms of modern wind turbines. Althogh there has been a lot of progress in determining the optimum $\epsilon$-parameter and avoiding too high loadings near the tip when employing crude mesh resolutions, there is still an urgent need for deriving a consistent technique to determine mesh-independent solutions of the ALM. The aim of the present work is develop and validate such a technique.

The model we propose is based on the analogy there exists between the actuator line model and the inviscid lifting line model. In the lifting line model the wing is represented by a line consisting of a distribution of bound vortices. Each change in the strenght of the bound vortices results in the formation of a free trailing vortex, which is convected downstream by the local velocity field in the wake. In the lifting line model the free vortices induce a velocity field on the wing that alters the inflow and which ensures a smooth distribution of the circulation near the tip that eventually goes to zero at the very tip. Ideally, a solution to the Euler or Navier-Stokes equations employing the ALM should result in the same circulation distribution and wake vortex system as the lifting line model. However, the size of the smoothing kernel in the filtering function dictates the minimum resolution of the vortex kernel, and acts in principle as a viscous inner core. This modifies the induction of the wake vortices in the ALM as compared to the same wake obtained using a lifting line technique. The technique that we propose is a relatively simple correction in the induced angle of attack to compensate for the added influence of the viscous core. Although the correction is a method for correcting the additional induction of the smoothing-kernel, we refer to it as a tip correction, as it is at the tip that it is most pronounced. 
The paper is structured as follows. In section 2 we briefly introduce to the ALM and show some results of computations without employing any corrections or by using directly the Prandtl tip correction. Next, in section 3 , we introduce the proposed correction technique. In section 4 we show some results for both planar wings and rotors to validate the method. Finally, in section 5 we conclude the work.

\section{NUMERICAL METHODS}

\subsection{Large Eddy Simulation Tool}

The numerical tool used in this study is an incompressible pseudo-spectral Large Eddy Simulation (LES) solver that has recently been developed at DTU Wind Energy [9]. The code uses Fourier series to discretize the horizontal directions, and a second-order central finite difference scheme in the vertical direction. The pseudo-spectral computation of the non-linear terms in the momentum equations are fully dealiased with a zero-padding operation. For the time integration, a second-order Adams-Bashforth scheme is used with a pressure-splitting technique, where the momentum equations are used to advance in time with the pressure field from the previous time step. The Poisson equation is subsequently solved directly to obtain the new pressure to project the velocities to a divergence free field. For the sub-grid-scale (SGS) modeling, a Smagorinsky model with a fixed constant of $C_{s}=0.18$ is employed for the current study. Since Fourier series enforce periodicity in the spanwise and streamwise directions, a buffer zone is used to gradually adjust the streamwise outgoing velocity to the desired, uniform, inflow condition. The length of the buffer zone is selected as $15 \%$ of the streamwise domain length. For the bottom and top boundaries, a stress-free condition is used. The forcing term from the wind turbine rotor is added as an additional term to the the momentum equations as

$$
\frac{\partial \boldsymbol{U}}{\partial t}=R H S+\boldsymbol{f}_{\boldsymbol{b}}
$$

$R H S$ here represents the advective, diffusion and pressure terms in the momentum equations and $f_{b}$ is the body force obtained with ALM. The calculation of this term is detailed below. For more details about the code, we refer to the PhD dissertation of Dag [9].

\subsection{The Actuator Line Technique}

In ALM, the turbine blades are represented as distributed body forces on a line that is assumed to be located at the quarter-chord line of the blade, as illustrated in Fig.1. The velocity of the overall flow field is described in a global coordinate system, $(x, y, z)$, with the origin located at the center of the wind turbine rotor, the $x$-axis pointing downstream along the axis of the rotor, and the $y$ - and $z$-axes perpendicular to this, as illustrated in Fig. 1 . With the $z$-axis pointing upward in the vertical direction, an angle $\theta$ defines the azimuthal position of one of the blades, with the three blades located an angle $\Delta \theta=2 \pi / 3$ from each other. To determine the relative velocity, as it is seen from the rotor blade, a local co-rotating coordinate system $(r, \theta, x)$ is utilized. This coordinate system is located in the rotor plane at a radial distance, $r$, from the rotor axis, with the $x$-axis pointing in the same direction as in the global system and with $\theta$ defining the azimuthal coordinate direction. To calculate the body forces, tabulated airfoil data and the angle of attack are used in a blade-element approach. First, the velocities from the flow solver, are interpolated to the velocity values on the actuator line points, referred to as $\left(u_{x}, u_{y}, u_{z}\right)$. With $\Omega$ denoting the rotational speed of the rotor, $r$ the blade radius, the local relative azimuthal velocity of the blade is given as

$$
u_{\theta}=\Omega r-\left(u_{y} \cos (\theta)+u_{z} \sin (\theta)\right)
$$

Wind Energ. 2017; 00:1-14 (C) 2017 John Wiley \& Sons, Ltd. 

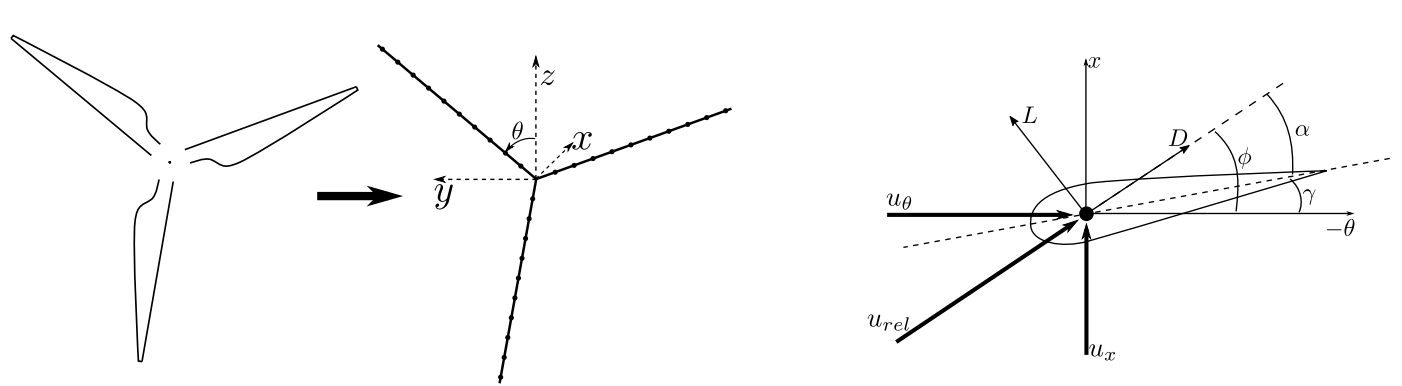

Figure 1. Illustration of the actuator line model (left), and local angles of a blade cross-section (right).

Note that, for simplicity, the rotor plane is assumed to be perpendicular to the flow. The flow angle for each actuator line point is obtained as

$$
\phi=\tan ^{-1}\left(u_{x} / u_{\theta}\right)
$$

which, together with the local twist and the pitch angle of the blade $(\gamma)$ is used, to obtain angle of attack values $(\alpha)$ for each actuator line point

$$
\alpha=\phi-\gamma
$$

The lift and drag forces for unit span is obtained with

$$
\begin{aligned}
& f_{L}=\frac{1}{2} \rho u_{r e l}^{2} c C_{L}, \\
& f_{D}=\frac{1}{2} \rho u_{r e l}^{2} c C_{D},
\end{aligned}
$$

where $u_{r e l}=\sqrt{u_{\theta}^{2}+u_{x}^{2}}$ is the local relative velocity, $c$ is the chord length and $C_{L}$ and $C_{D}$ are the local lift and the drag coefficients, respectively. At this stage, these forces are projected to tangential and normal components by using the local flow angles as

$$
\begin{aligned}
& f_{\theta}=f_{L} \sin (\phi)-f_{D} \cos (\phi), \\
& f_{n}=f_{L} \cos (\phi)+f_{D} \sin (\phi) .
\end{aligned}
$$

Then in grid coordinates the forces read

$$
\begin{array}{r}
f_{x}=-f_{n}, \\
f_{y}=-f_{\theta} \cos (\theta), \\
f_{z}=f_{\theta} \sin (\theta) .
\end{array}
$$

Applying these forces directly to the closest CFD grid point would produce discontinuities. To avoid this, a filtering procedure is applied by using a 3D Gaussian filtering function which is a function of the distance $d$ that is determined individually for each grid-actuator line point couple as

$$
d_{i, j, k}^{N, m}=\sqrt{\left(x_{i, j, k}-x^{N, m}\right)^{2}+\left(y_{i, j, k}-y^{N, m}\right)^{2}+\left(z_{i, j, k}-z^{N, m}\right)^{2}}
$$

Here $(i, j, k)$ are indices that refer to the $(x, y, z)$ axes in the global coordinate system, and superscripts $N$ and $m$ represent the blade and actuator line point indices respectively. The 3D Gaussian filter kernel reads

$$
\eta_{i, j, k}^{N, m}=\frac{1}{\epsilon^{3} \pi^{\frac{3}{2}}} \exp \left[-\left(\frac{d_{i, j, k}^{N, m}}{\epsilon}\right)^{2}\right] .
$$


With the known kernel,the body forces $\boldsymbol{f}_{\boldsymbol{b}}$ in the momentum equations are determined as

$$
\boldsymbol{f}_{\boldsymbol{b}(\boldsymbol{i}, \boldsymbol{j}, \boldsymbol{k})}=\sum_{N} \sum_{m} \eta_{i, j, k}^{N, m}\left[\begin{array}{l}
f_{x}^{N, m} \\
f_{y}^{N, m} \\
f_{z}^{N, m}
\end{array}\right] .
$$

\subsection{Results using present formulation of the ALM}

To demonstrate the inadequacy of using a coarse mesh in the ALM, blade loading distributions of the NREL Phase VI rotor is computed for a steady flow case with a rotor resolution of 10 mesh points along the rotor radius. It should be noted that, as a general guideline, the minimum resolution of the wing should be around 30-50 grid points along the rotor radius. The main geometry and operational conditions of the rotor are given in Table I. The size of the employed domain

Table I. Operation conditions for the NREL Phase VI rotor

$\begin{array}{ll}\text { Rotor diameter } & 10.058 \mathrm{~m} \\ \text { Root cut out } & 0.9 \mathrm{~m} \\ \text { Rotational speed } & 7.50107 \mathrm{rad} / \mathrm{s} \\ \text { Pitch angle } & 3^{\circ} \\ \text { Cone angle } & 0^{\circ} \\ \text { Wind speed } & 7 \mathrm{~m} / \mathrm{s}\end{array}$

is $\left[L_{x}, L_{y}, L_{z}\right]=180 \times 60 \times 60 \mathrm{~m}$. The grid resolution is $\Delta=\Delta x=\Delta y=0.5 \mathrm{~m}$ and $\Delta z=0.25 \mathrm{~m}$, with $x$ and $y$ being the axes parallel to the ground, and $z$ the vertical axis. With this grid setting, a filter kernel $\epsilon=3 \Delta=1.5 \mathrm{~m}$ is used for the actuator line forces. The resulting normal and tangential loading distributions are shown in Fig. 2. To demonstrate the problems of representing correctly the tip loading when using coarse meshes, the ALM computations are compared to results from a similar blade-element momentum (BEM) computation and an ALM computation using the Prandtl tip corection. As seen in the figure, at the inner parts of the blade, the loadings from BEM and ALM match well. However, at the tip region an overestimation of the ALM loadings is clearly visible. Furthermore, the loading do not tend to zero at the very tip. Correcting the load distribution by the Prandtl tip correction, however, does not the remedy the situation, as the loading becomes highly underestimated. As will be shown later, this problem only occurs when using a coarse mesh. However, it is not always possible to carry out computations on sufficiently fine meshes, and there is obviously a need for a technique that may produce accurate results on coarse meshes.
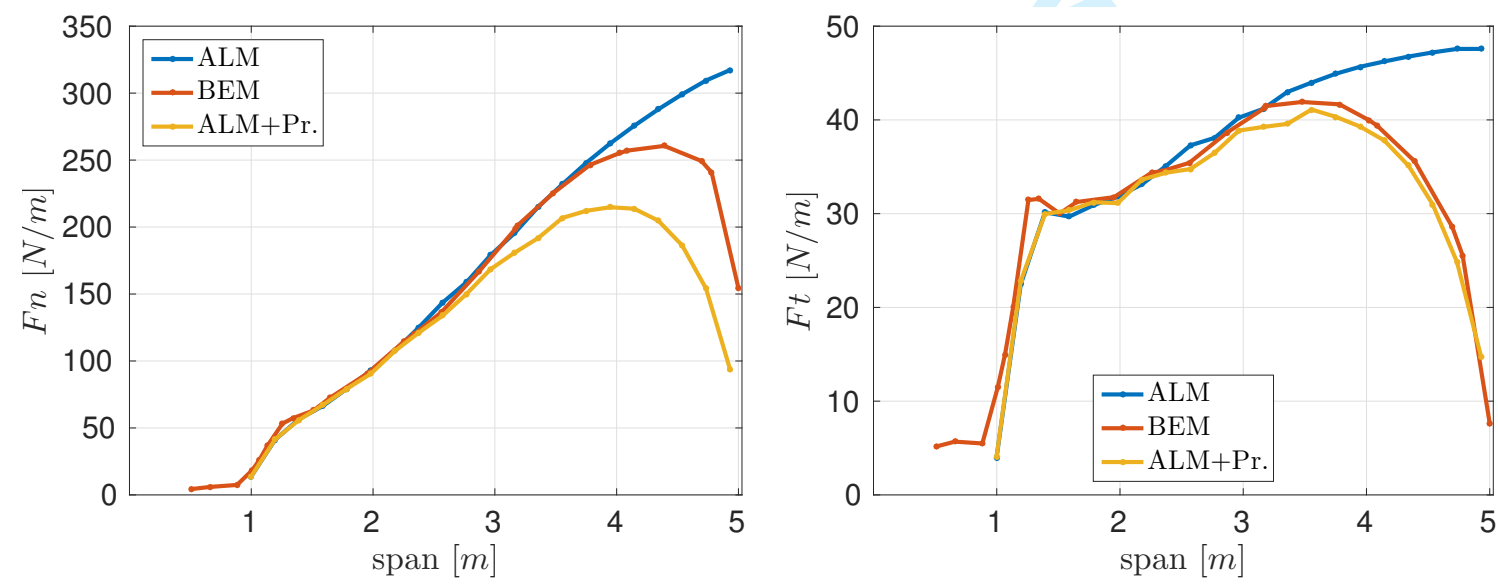

Figure 2. Comparison of loading distributions from BEM and ALM and ALM with Prandtl's correction for NREL Phase VI rotor operating under $7 \mathrm{~m} / \mathrm{s}$ steady inflow

Wind Energ. 2017; 00:1-14 (C) 2017 John Wiley \& Sons, Ltd. 


\subsection{Influence of the kernel size}

To further investigate the over-shooting issue of the ALM, we here simplify the case and apply the ALM for a non-rotating rectangular wing. To have a reference circulation distribution, the lifting line technique (LLT) is used for the same wing and results are compared with those obtained from ALM with different kernel sizes of the filtering function. The rectangular wing used in this study has a constant chord length of $1 \mathrm{~m}$ with a span length of $10 \mathrm{~m}$. The wing is positioned at a geometrical angle of attack of $5^{\circ}$, and the incoming velocity is fixed at $8 \mathrm{~m} / \mathrm{s}$. Inviscid airfoil data of the NACA 0012 airfoil is used for both ALM and LLT. For the ALM computations, different $\epsilon$-values are used together with a domain size of $50 \times 30 \times 20 \mathrm{~m}$, with a varying grid resolution and a fixed kernel value $\epsilon / \Delta=2$. A comparison of the circulation distributions is presented below in Fig.3. Due to symmetry, only the half-span is shown in the plot. In the LLT case, due to

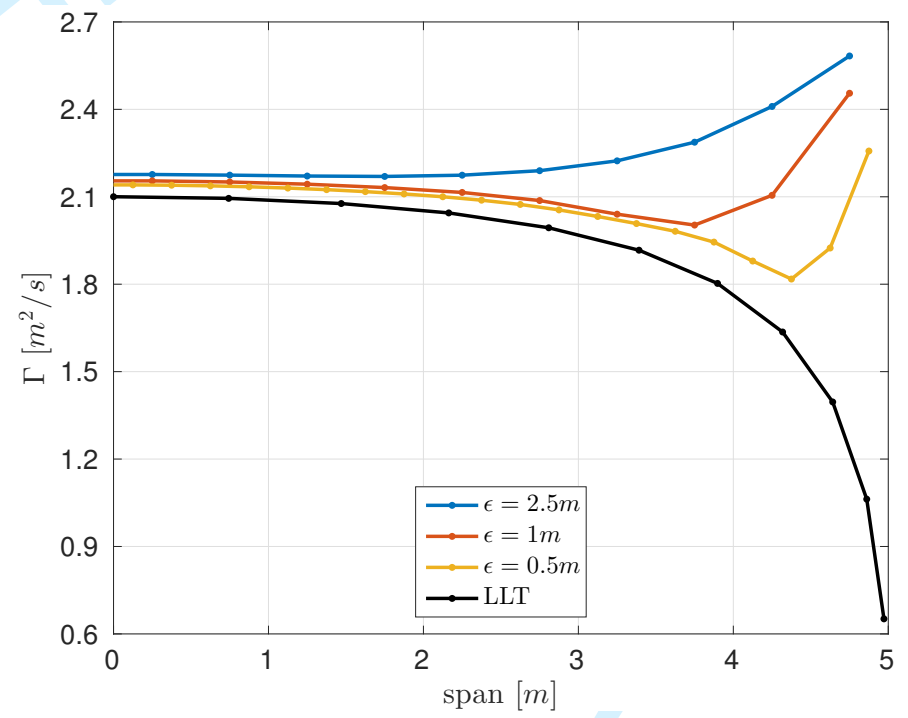

Figure 3. Comparison of circulation distributions from LLT and ALM with different $\epsilon$ values for $A R=10$ wing

the strong tip vortices, the induction goes to infinity and circulation tends to zero at the very tip of the wing. This is not the case for the ALM where the circulation for all $\epsilon$-values is seen to increase near the tip. On the other hand, with decreased $\epsilon$-values, the ALM circulation levels converge towards the LLT results, yet with a peak at the tip region. This behavior can only be explained by an underestimated wake induction near the tip, causing an increased angle of attack, which results in a stronger circulation.

In order to clarify the effect of the $\epsilon$-parameter on the bound vortices, an infinitely long wing with constant circulations is employed as model example. In this case the bound vortex generated by the ALM can be studied without being disturbed by the influence of the free vortices. The outcome of this is shown in Fig. 4, where the computed normal velocity distribution is shown for different $\epsilon / \Delta$-values. What is seen here is esentially the velocity distribution induced by a two-dimensional vortex which is being filtered by the Gaussian filter function. A high $\epsilon$-value tends to smear out the velocity whereas a small $\epsilon$-value results in a more distinct velocity distribution, tending towards the inviscid value of $\Gamma / 2 \pi r$. An important part of the ALM is to determine the local angle of attack as this forms the input to the airfoil data. Since the angle of attack is determined from the local velocity, as shown in Eqs. 1-3, the induction from a vortex with a high $\epsilon$-value tends to overpredict it, with the result that the loadings become overestimated. It should be emphasized that it is not the induction of 


\section{CORRECTION METHODOLOGY}

To account for the missing induction, it is noted that the Lamb-Oseen vortex model ([11]) is equal to the employed filtering function and results in the exact same induction as the one obtained from an ALM computation with viscous core size corresponding to the size of the smoothing-kernel $\epsilon$. The analytic formulation of a semi-infinite Lamb-Oseen vortex model is given as,

$$
w_{i}=\frac{\Gamma}{4 \pi r}\left[1-\exp \left[-\left(\frac{r}{r_{v c}}\right)^{2}\right]\right],
$$

where $\Gamma$ is the vortex strength and $r_{v c}$ is the viscous core size. From this expression it is readily seen that the first term corresponds to the inviscid part of the induction and the second term accounts for the influence of the viscous core. In Fig. 5 it is shown that, by replacing $r_{v c}$ with the $\epsilon$-values used in an ALM computation, the vortex model gives the exactly same distribution of induced velocities.

Hence, by subtracting the viscous part from the induction of the Lamb-Oseen model, one may establish a model for correcting the unwanted influence of the viscous core on the induced velocity,

$$
w_{\text {corr }}=\frac{\Gamma}{4 \pi r} \exp \left[-\left(\frac{r}{\epsilon}\right)^{2}\right]
$$

As the bound vortex does not contribute to the induced velocity defining the angle of attack (the angle of attack is essentially determined at the center of the bound vortex), the correction is applied on the induction from the trailing 


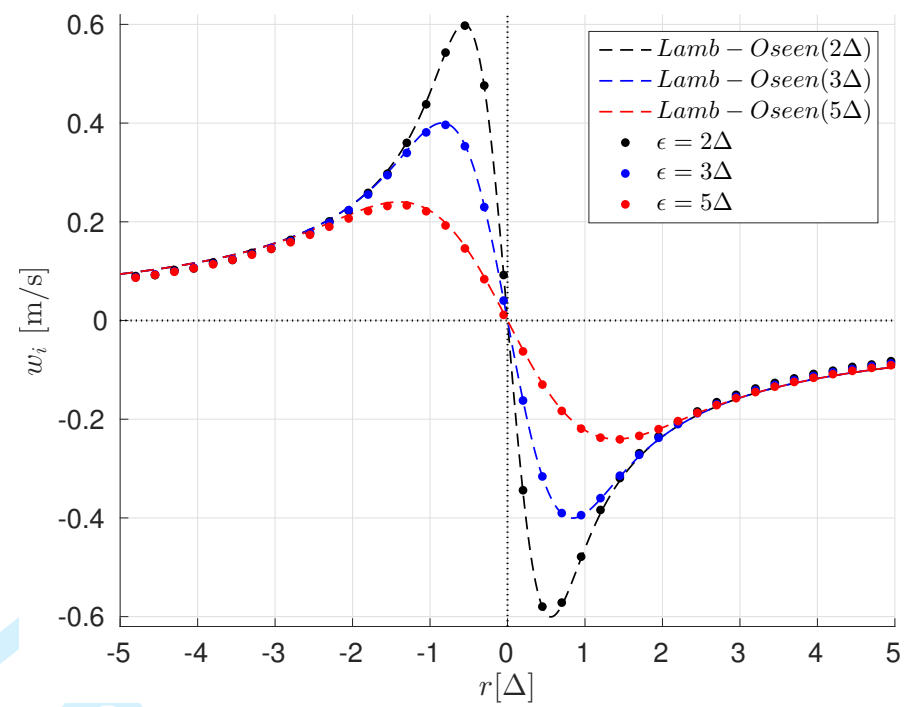

Figure 5. Comparison of Lamb-Oseen vortex model with induction levels obtained from CFD computation with given $\epsilon$-parameter.

vortices in the wake, which then corrects the induced velocity through the Biot-Savart induction law. However, to do this it is required to know the position and induction of the wake vortices.

\subsection{Translating planar wing}

To demonstrate how the correction works, we here first apply it on a translating planar wing. The correction is meant to be applied at the angle of attack calculation stage, where the total additional induction from the wake system, $w_{\text {corr }}$, is added to the local relative velocity and angle of attack value is determined. To apply the correction, the location of the wake vortices and their strengths have to be defined in advance as these are required, see Eq. 16. For a translating planar wing application, wake vortices can be assumed to form a straight vortex sheet which is parallel to the flow and which trail from the quarter-chord line of the wing (see the Fig.6). Then, for each actuator line point, the total correction from the viscous

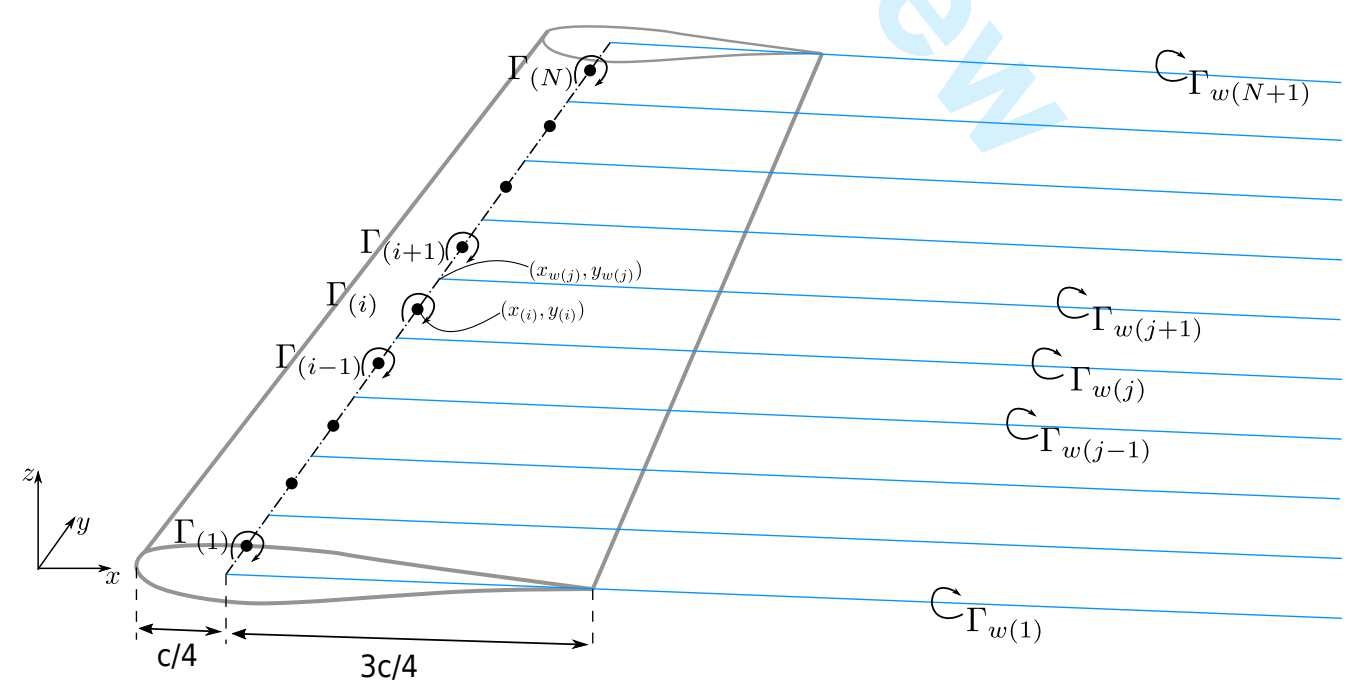

Figure 6. Illustration of a vortex system of a translating planar wing 
cores of the wake vortices can be calculated as

$$
w_{\operatorname{corr}(i)}=\sum_{j=1}^{N+1} \frac{\Gamma_{w(j)}}{4 \pi d_{(i, j)}} \exp \left[-\left(\frac{d_{(i, j)}}{\epsilon}\right)^{2}\right]
$$

Here $N$ denotes the total number of actuator line points on and $\Gamma_{w}$ represents the circulation strength of the wake vortices, with magnitudes equal to the difference between two neighboring bound circulations (see Fig.6),

$$
\Gamma_{w(j)}=\Gamma_{(j)}-\Gamma_{(j-1)}
$$

For the tip vortices, the magnitudes are equal to the bound circulations which are closest to the tips. In this system, the strength of a bound circulation is a function of the corresponding chord, $c$, relative velocity, $u_{r e l}$, and the lift coefficient, $C_{L}$. Employing the Kutta-Joukowsky theorem, we get

$$
\Gamma_{(i)}=\frac{1}{2} c_{(i)} C_{L(i)} u_{r e l(i)}
$$

In Eq.17, the variable $d$ is defined individually for each trail-actuator line point couple. The definition in the given coordinate system * (see Fig.6) can be written as

$$
d_{(i, j)}=y_{(i)}-y_{w(j)}
$$

Knowing the total correction for the induced velocity, the resulting corrected angle of attack is determined as,

$$
\alpha_{(i)}=\alpha_{g}-\sin ^{-1}\left(\frac{-u_{z(i)}+w_{\operatorname{corr}(i)}}{u_{\operatorname{rel}(i)}}\right) .
$$

In Eq. 21, $\alpha_{g}$ represents the geometrical angle of attack of the wing, and $u_{r e l}$ and $u_{z}$ are the relative and the vertical velocity components, respectively. An illustration of the induction correction for a single vortex line is shown in Fig. 7. In the figure, the dashed blue line represents the underestimated induction from the tip vortex using AL / CFD, which causes too large tip loadings. The thick blue line is the induction that would occur in a LLT case. Hence, the red swept area is the induction correction, which corrects the angle of attack values at the region. The consequence of modifying the values of angle of attack, bound vorticity and, hence, the strengths of the wake vortices change as a function of time. Therefore, the correction requires an iterative procedure in each time step of a CFD computation.

\subsection{Wind turbine rotor}

The correction procedure for a turbine rotor proceeds in principle as the one for a translating planar wing. The only difference is due to the wake structure, which adds a level of complexity to the calculation. Due to the three-dimensional formation of the wake (see Fig. 8 (left)), it is needed to define a discrete vortex lines and calculate the induction correction in all three space directions. By using vector notation, the induction correction from a vortex line is written as

$$
\left[\begin{array}{l}
w_{\text {xcorr }} \\
w_{\text {ycorr }} \\
w_{z \text { corr }}
\end{array}\right]=\frac{\Gamma}{4 \pi} \frac{\overrightarrow{d l} \times \vec{d}}{|\vec{d}|^{3}}\left[\exp \left[-\left(\frac{|\vec{d}|}{\epsilon}\right)^{2}\right]\right]
$$

where $\overrightarrow{d l}$ is the vector representing the direction and the length of the vortex line, $\vec{d}$ is the vector from the center of the vortex line to the induction calculation point (see Fig. 8 (right)). Then for each actuator line point the total induction

${ }^{*}$ Note that span of the wing is aligned with the $y$ axis for simplicity. 


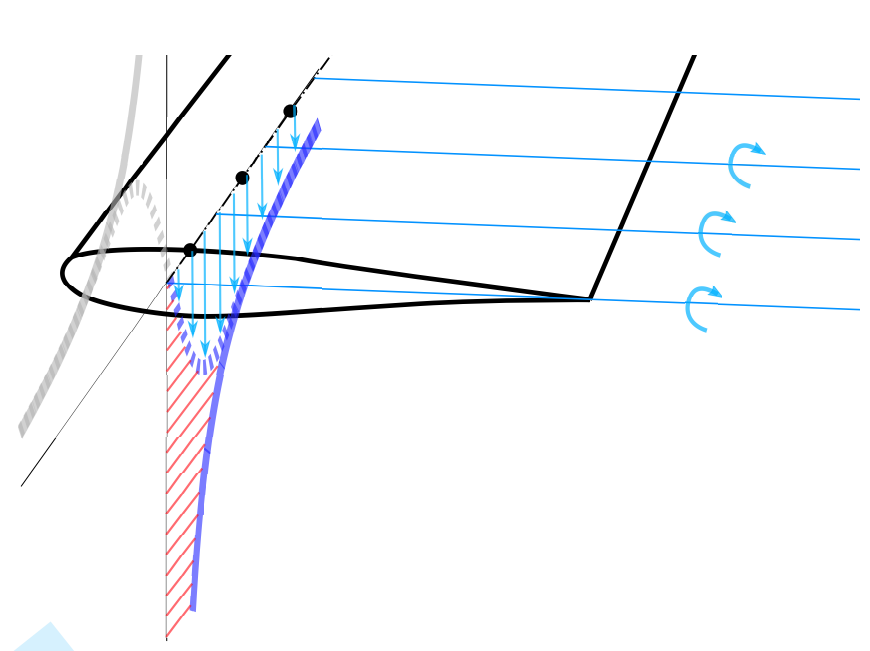

Figure 7. Illustration of the induction correction for the tip vortex

correction can be calculated by summing up all the corrections from the wake system as

$$
\left[\begin{array}{l}
w_{\text {xcorr }} \\
w_{y \text { corr }} \\
w_{z \text { corr }}
\end{array}\right]^{N, m}=\sum_{h} \sum_{p} \sum_{q} \frac{\Gamma_{w(h, p, q)}}{4 \pi} \frac{\vec{d}_{h, p, q} \times \vec{d}_{h, p, q}^{N, m}}{\left|\vec{d}_{h, m, q}^{N, m}\right|^{3}}\left[\exp \left[-\left(\frac{\left|\vec{d}_{h, p, q}^{N, m}\right|}{\epsilon}\right)^{2}\right]\right] .
$$

Here, the indices $h, p$ and $q$ represent the blade number, span position and the azimuthal position, respectively, for the wake vortices, and $N$ and $m$ represent the blade number and span position for actuator line points, respectively (see Fig. 8 (right)). The circulation strengths are calculated similar to the planar case as

$$
\Gamma^{N, m}=\frac{1}{2} c^{N, m} C_{L}^{N, m} u_{r e l}^{N, m}
$$

and

$$
\Gamma_{w(h, p, q)}=\Gamma_{(p-1, q)}-\Gamma_{(p, q)} .
$$

With the calculated total induction correction for each actuator line point, the flow angle can be corrected as

$$
\phi^{N, m}=\tan ^{-1}\left(\frac{u_{x}^{N, m}+w_{x c o r r}^{N, m}}{u_{\theta}^{N, m}+w_{\theta \text { corr }}^{N, m}}\right),
$$

where

$$
w_{\theta \text { corr }}^{N, m}=w_{y \operatorname{corr}}^{N, m} \cos \left(\theta^{N}\right)+w_{z \operatorname{corr}}^{N, m} \sin \left(\theta^{N}\right)
$$

Next, the new angle of attack values can be computed locally by Eq.4.

To generate the discrete wake, the helical pitch of the each individual trailing vortex is assumed to be constant and equal to the relative flow angle at the position where the trailing vortex is released. Since the flow angle is updated in every time-step, depending of the local flow conditions, this gives a quasi-steady behavior for the correction. In this work, the length of the helical vortex sheet is fixed at two full revolutions, and the azimuthal discretization of the wake is made with $2^{\circ}$ intervals. 

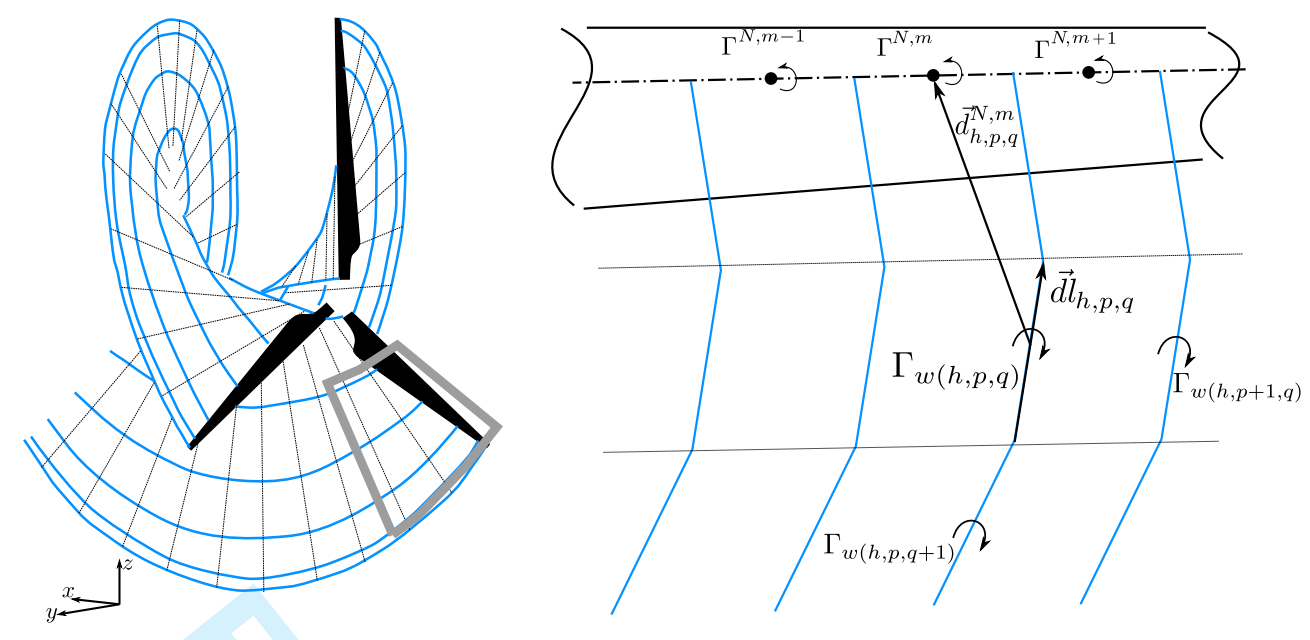

Figure 8. Illustration of the helical wake released from a wind turbine rotor(left), a close-up illustration of the wake formation of the single blade(right).

\section{RESULTS}

\subsection{Translating planar wing}

To validate the new correction, we first compute the flow past a translating rectangular wing with a constant chord $c=1$ $\mathrm{m}$ and aspect ratios 6 and 10. For the ALM computations, a CFD grid resolution of $100 \times 60 \times 80$ is used. This resolution corresponds to $\Delta=0.5 \mathrm{~m}$ in the span-wise and stream-wise directions, and $0.25 \mathrm{~m}$ in the vertical direction. The filter parameter, $\epsilon$, is taken as a chord length, which corresponds to $2 \Delta$ for any given grid resolution. The inflow velocity is fixed at $8 \mathrm{~m} / \mathrm{s}$ and the geometrical angle of attack for both wings is $5^{\circ}$. In Fig. 9, circulation distributions are plotted for both wings and compared against LLT computations. In the figure, the corrected ALM computations are shown as ALM*, and, due to symmetry, only half-span distributions are shown.

As seen from the figure, the overestimation of the tip circulations is visible in the ALM simulations, with a peak value that is higher than the maximum circulation occurring at the middle section of the wing. Applying the new correction fixes the issue and provides comparable results with LLT. Additionally, it is important to note that it is not only at the tip region, but also at the mid-regions, that the correction provides circulation levels similar to LLT computations.

\subsection{Wind turbine rotor}

For rotor applications, in order to cover a wider range of possibilities with a minimum of computations, two different rotors are employed, namely the NREL Phase VI and the NREL 5MW rotor. The two-bladed Phase VI rotor runs at a tip speed ratio of 5.39 and has an averaged aspect ratio of 7.5 , whereas the $5 \mathrm{MW}$ rotor is a three-bladed type operating at a higher tip speed ratio of 7.55 , with slender blades of an average aspect ratio of 15.8 . The operating conditions of the rotors are detailed in Table II.

For this study we have conducted 8 simulation cases for each rotor. For the Phase VI rotor, a domain size of $180 \times 60 \times 60 \mathrm{~m}$ is used and the rotor center is positioned at $20 \times 30 \times 30 \mathrm{~m}$. For the $5 \mathrm{MW}$ rotor, a domain size of $1512 \times 504 \times 504 \mathrm{~m}$ is employed and the rotor center is positioned at $252 \times 252 \times 252 \mathrm{~m}$. For both domains, the blockage effects are checked and it is concluded that the current domain sizes are sufficient. Further details of the CFD cases are provided in Table III. In the table, $\Delta t$ represents the time step size in the CFD computations, which is restricted to be smaller than the time it takes for a blade tip to travel one grid spacing. The total number of time steps for each 


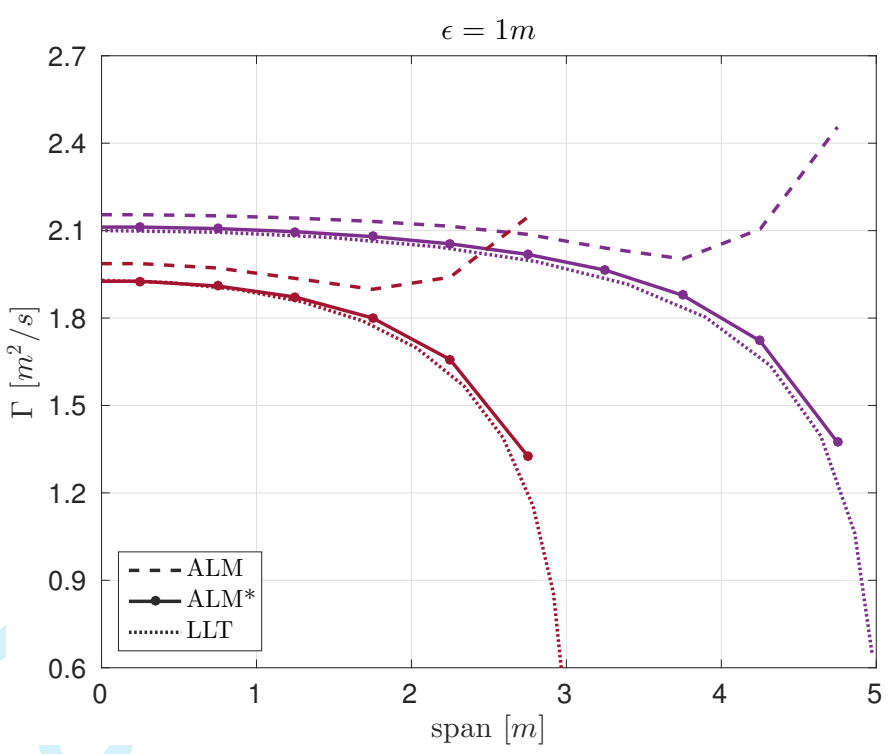

Figure 9. Comparison of circulation distribution obtained from ALM, corrected ALM (ALM*) and LLT computations for translating planar wings with $A R=6,10$ 


की
$180 \times 60 \times 120$
$180 \times 60 \times 120$
$180 \times 60 \times 120$
$180 \times 60 \times 120$
$720 \times 240 \times 480$
$720 \times 240 \times 480$
$720 \times 240 \times 480$
$720 \times 240 \times 480$
$120 \times 40 \times 80$
$120 \times 40 \times 80$
$120 \times 40 \times 80$
$120 \times 40 \times 80$
$480 \times 160 \times 320$
$480 \times 160 \times 320$
$480 \times 160 \times 320$
$480 \times 160 \times 320$

\begin{tabular}{|c|c|c|}
\hline$\nabla$ & $<$ & s) \\
\hline 2 & 5 & 6 \\
\hline & & \\
\hline & 5 & 10 \\
\hline & 5 & 10 \\
\hline 0.5 & 20 & 1.5 \\
\hline 0.5 & 20 & 1.5 \\
\hline 0.5 & 20 & 2.5 \\
\hline 0.5 & 20 & 2.5 \\
\hline 12.6 & 5 & 37.8 \\
\hline 12.6 & 5 & 37.8 \\
\hline 12.6 & 5 & 63 \\
\hline 12.6 & 5 & 63 \\
\hline 3.15 & 20 & 9.45 \\
\hline 3.15 & 20 & 9.45 \\
\hline 3.15 & 20 & 15.7 \\
\hline & & \\
\hline
\end{tabular}

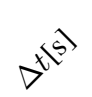

4.3E-2

4.3E-2

4.3E- 2

4.3E- 2

2E-3

2E-3

2E-3

2E-3

$3.9 \mathrm{E}-2$

$3.9 \mathrm{E}-2$

$3.9 \mathrm{E}-2$

3.9E-2

$1.3 \mathrm{E}-2$

$1.3 \mathrm{E}-2$

$1.3 \mathrm{E}-2$

$1.3 \mathrm{E}-2$

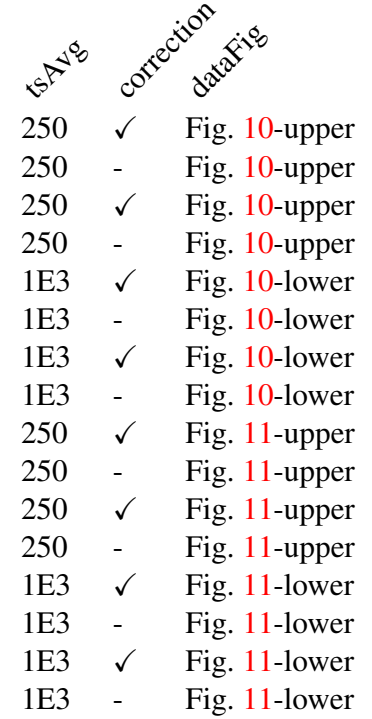

Table III. Details of the CFD cases for rotor simulations

\section{DISCUSSION AND CONCLUSIONS}

The projection function that was introduced to smear out the body forces in the actuator line method (ALM) alters the induced velocities, which in most cases results in too high blade loadings. This is in particular pronounced near the tip where strong vortices are trailed from the blade. To overcome this problem, a correction model has been introduced and tested. The idea behind the model is to correct the underestimated wake induction on the control points of the actuator line. By using the Biot-Savart induction law together with the prescribed position of the wake vortices, the influence of the projection function on the resulting induced velocity is removed. This approach results in a correction technique to correct the angle of attack, which subsequently reduces the blade loadings. The new correction is first applied on a translating planar wing, showing that it works excellent for this simple flow case. Next, the NREL Phase VI and 5MW rotors were employed to test the correction. For both rotors, the correction showed great improvement in blade loadings even for CFD grid resolutions as low as 5 grid points per rotor radius. Applying the correction on ALM computations of the Phase VI rotor resulted in load levels very close to the results obtained from blade element momentum (BEM) computations, although a too crude resolution may result in minor deviations of the tangential loads. For both turbines, it is seen that a CFD resolution corresponding to 20 grid points per rotor radius is sufficient to obtain results which are in excellent agreement with BEM computations for both normal and tangential loadings. It can be concluded that the application of this new correction results in very convincing results that indeed reduces the loadings to the anticipated level for both translating planar wings and for wind turbine rotor configurations even with low CFD grid resolutions.

\section{REFERENCES}

1. J. N. Sørensen and W. Shen, "Numerical modeling of wind turbine wakes," Journal of Fluids Engineering, 2002.

2. N. Troldborg, Actuator Line Modeling of Wind Turbine Wakes. PhD thesis, DTU, 2008. 

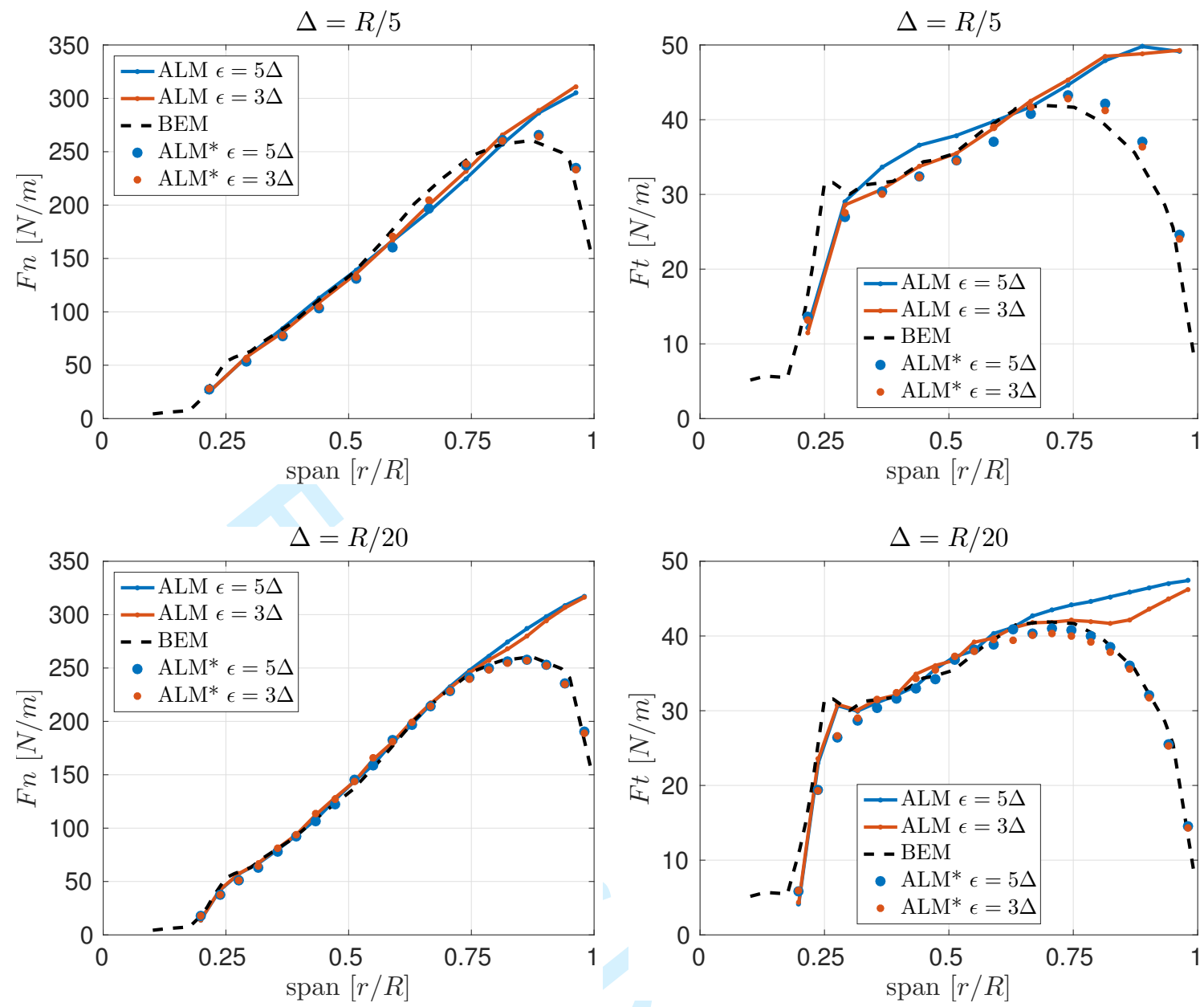

Figure 10. Comparison of normal and tangential loadings from ALM with and without the correction, and BEM computations for NREL Phase VI turbine.

3. P. K. Jha, M. J. Churchfield, P. J. Moriarty, and S. Schmitz, "Guidelines for volume force distrubutions within actuator line modeling of wind tturbine on large-eddy simulation-type grids," Journal of Solar Energy Engineering, 2014.

4. M. Shives and C. Crawford, "Mesh and load distribution requirements for actuator line cfd simulations," Wind Energy, 2013.

5. L. A. Martínez-Tossas, M. J. Churchfield, and C. Meneveau, "Optimal smoothing length scale for actuator line models of wind turbine blades," Wind Energy, 2017.

6. J. N. Sørensen, General Momentum Theory for Horizontal Axis Wind Turbines. Springer, 2016.

7. W. Z. Shen, J. N. Sørensen, and R. Mikkelsen, “Tip loss correction for actuator/navier-stokes computations,” Journal of Solar Energy Engineering, 2005.

8. J. N. Sørensen, K. O. Dag, and N. R. García, “A refined tip correction based on decambering,” Wind Energy, 2016.

9. K. O. Dag, Combined pseudo-spectral / actuator line model for wind turbine applications. PhD thesis, Technical University of Denmark Department of Wind Energy, May 2017.

10. W. Z. Shen, H. H. Zhang, and J. N. Sørensen, "The actuator surface model: A new navier-stokes based model for rotor computations," Journal of Solar Energy Engineering, 2009.

11. H. Lamb, Hydrodynamics, 6th ed. Cambridge University Press, 1932. 

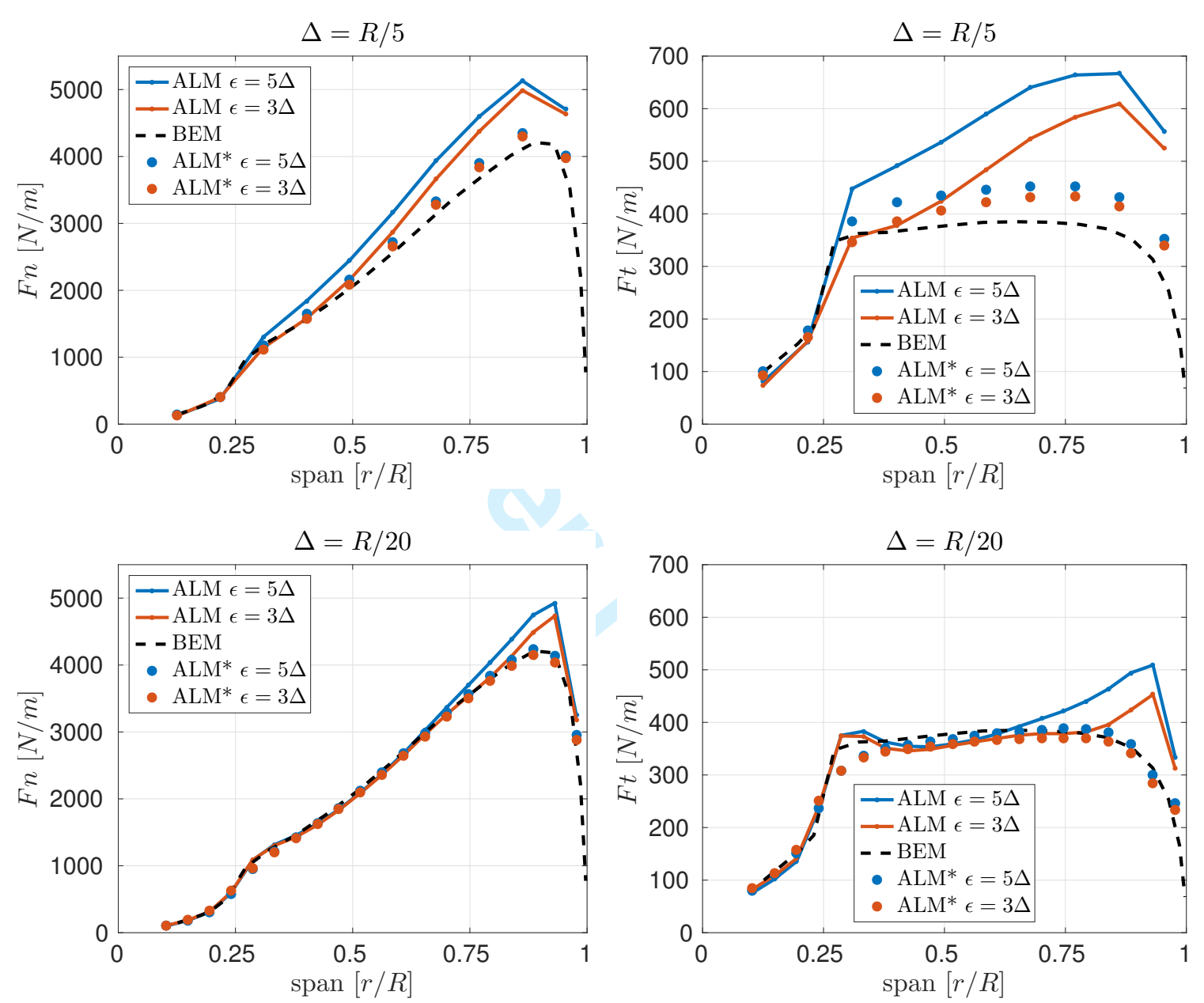

Figure 11. Comparison of normal and tangential loadings from ALM with and without the correction, and BEM computations for NREL $5 \mathrm{MW}$ turbine. 